\title{
Determining the properties of dark matter halos from gravitational lensing measurements in galaxy clusters
}

\section{Leandro J. Beraldo e Silva ${ }^{* a, c}$, Marcos Lima ${ }^{a}$ and Laerte Sodré Jr. ${ }^{b}$}

${ }^{a}$ Departamento de Física Matemática, Instituto de Física, Universidade de São Paulo, São Paulo SP, Brazil

${ }^{b}$ Departamento de Astronomia, Instituto de Astronomia, Geofísica e Ciências Atmosféricas, Universidade de São Paulo, São Paulo SP, Brazil

${ }^{c}$ CAPES Foundation, Ministry of Education of Brazil, Brasília - DF 70.040-020, Brazil

E-mail: Mberaldodif.usp.br mLimadema.if.usp.br

laertedastro.iag.usp.br

\begin{abstract}
We use the stacked gravitational lensing mass profile of four high-mass $\left(M \gtrsim 10^{15} M_{\odot}\right)$ galaxy clusters around $z \approx 0.3$ from [四] to fit dark matter density profiles of phenomenological and theoretical models. The best overall fit is obtained with the phenomenological NFW profile. Among the theoretically predicted models, the DARKexp represents the best fit, with performance close to that of the best phenomenological ones. We conclude that DARKexp model retains important features of nearly universal density profiles of self-gravitational systems, and may provide a dynamical basis for the phenomenology of these systems.
\end{abstract}

VIII International Workshop on the Dark Side of the Universe

June 10-15, 2012

Búzios, Rio de Janeiro, Brasil

\footnotetext{
*Speaker.
} 


\section{Introduction}

Since the studies of Zwicky [2] on the internal kinematics of Coma cluster, dark matter is known to represent the main massive component of astrophysical objects such as galaxies and galaxy clusters. Evidences for this massive component include the rotation curves of spiral galax-

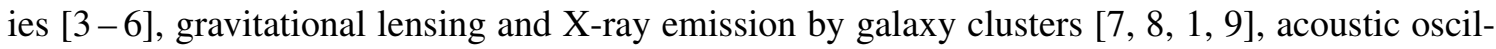
lations measured in the cosmic microwave background [ए0] and studies of structure formation on numerical simulations [ए]].

In fact, simulations have indicated that these systems exhibit nearly universal density $\rho(r)$ [ए2] and pseudo phase-space $\rho(r) / \sigma^{3}(r)$ [[1]] profiles, where $\sigma(r)$ is either the radial or total velocity dispersion. Interestingly, these universal density profiles also seem to agree with observations. Therefore, since numerical simulations approximately provide the observed features of density profiles, one could think that the hypothesis assumed in performing the simulations are correct and there is not much else to be done. We argue, however, that a description of these systems in the spirit of statistical mechanics is necessary for obtaining a deeper dynamical basis of their phenomenology.

Since the pioneer work of Lynden-Bell [14]] on the violent relaxation process in gravitational systems, many first-principle models have been developed trying to explain the features seen in simulations and observations. In particular, there has been a great effort to make predictions of the three-dimensional density profile $\rho(\mathbf{r})$ of dark matter halos. The connection with observations is made via the surface density profile $\Sigma(R)$ projected in the plane perpendicular to the line of sight $r_{\|}$

$$
\Sigma(R)=\int d r_{\|} \rho\left(r_{\|}, R\right),
$$

where $\mathbf{r}=\left(r_{\|}, R\right)$ and $R$ is the projected distance on the plane of the sky. The surface mass density profile $\Sigma(R)$ can be inferred e.g. with gravitational lensing techniques, which do not require assumptions of hydrostatic equilibrium, as in dynamical methods. In practice, however, it is difficult to separate the purely gravitational effects related to dark matter from the dissipative effects due to baryonic matter. Thus, compared to galaxies, for which the dissipative effects of cold baryons are important, galaxy clusters are excellent to test the distribution of dark matter, because in clusters most of the baryons are hot and dissipate less. Thus, the total density profile provides reliable information about the dark matter density profile.

In this work we use the stacked surface density profile from four massive galaxy clusters with similar mass and redshift to test both phenomenological and theoretical models. In $\S \square$ we briefly describe the data used in this work. In $\S[$ and $\S$ 田 we present the models that we have tested. Our results are shown in $\$[$ and discussed in $\S$.

\section{Data}

We use the data of [四], who combined weak-lensing shear, magnification, and strong-lensing measurements of four high-mass $\left(M \gtrsim 10^{15} M_{\odot}\right)$ galaxy clusters (A1689, A1703, A370, C10024+17) with redshifts $z \approx 0.3$. The strong lensing data was based on Hubble Space Telescope observations for the central regions of those clusters and combined with previous weak lensing measures obtained by [ए6], extending to the outer regions $\left(R \lesssim 3.5 h^{-1} \mathrm{Mpc}\right)$ of the clusters. The surface 
density profiles of the four clusters were stacked in order to reduce the cosmic noise and smooth effects due to asphericity and substructures. The resulting data points are shown in Fig. $\mathrm{W}$.

\section{Phenomenological models}

A number of phenomenological models for density profiles of dark matter halos and galaxy clusters have been proposed as parameterized functions that fit reasonably well simulations and observations, with no regards to fundamental principles or theoretical motivation. We have tested six models in this class. Two of them have only scale parameters in density $\left(\rho_{s}\right)$ and distance $\left(r_{s}\right)$ : they are the NFW profile [12], proposed to fit $\Lambda \mathrm{CDM}$ simulations and the Hernquist profile [ए]], which gives analytical expressions for some dynamical quantities such as the energy distribution function. The other phenomenological models have an extra shape parameter in addition to the two scale parameters: the gNFW [1]8], a generalization of NFW with the inner slope $\alpha$ as a free

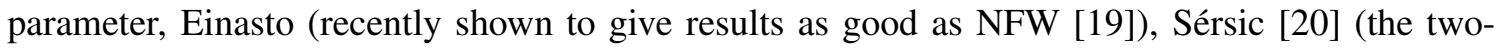
dimensional, original version of Einasto profile) and Stadel [ [2]]. The expressions for the density profiles of these models are shown in Eq. (B.]), except for the bi-dimensional Sérsic profile.

$$
\rho(r)= \begin{cases}\frac{\rho_{s}}{\left(r / r_{s}\right)\left(1+r / r_{s}\right)^{2}}, & \text { NFW } \\ \frac{\rho_{s}}{\left(r / r_{s}\right)^{\alpha}\left(1+r / r_{s}\right)^{3-\alpha}}, & \text { gNFW } \\ \rho_{s} \exp \left\{-2 n\left[\left(r / r_{s}\right)^{1 / n}-1\right]\right\} & \text { Einasto } \\ \rho_{s} \exp \left\{-\lambda\left[\ln \left(1+r / r_{s}\right)\right]^{2}\right\}, & \text { Stadel } \\ \frac{\rho_{s}}{\left(r / r_{s}\right)\left(1+r / r_{s}\right)^{3}}, & \text { Hernquist }\end{cases}
$$

The NFW and Hernquist models have analytic expressions for the surface mass density $\Sigma(R)$. The Sérsic model is defined in terms of $\Sigma(R)$ as

$$
\Sigma(R)=\Sigma_{e} \exp \left\{-b_{n}\left[\left(R / R_{e}\right)^{1 / n}-1\right]\right\} .
$$

All the phenomenological profiles described above are shown in Fig. $\square$.

\section{Theoretical models}

Some of the theoretical models we investigate here are based on the hypothesis of hydrostatic equilibrium between the gravitational attraction and the pressure $P(r)$ due to velocity dispersion in an isotropic distribution with total mass inside radius $r$ given by $M(r)$ :

$$
\begin{gathered}
\frac{d P}{d r}=-\rho(r) \frac{G M(r)}{r^{2}}, \\
M(r)=\int_{0}^{r} d r^{\prime} 4 \pi r^{\prime 2} \rho\left(r^{\prime}\right) .
\end{gathered}
$$


Combining Eqs. 4. 1 and 4.2 we have

$$
\frac{d}{d r}\left[\frac{r^{2}}{\rho(r)} \frac{d P}{d r}\right]=-4 \pi G r^{2} \rho(r) .
$$

Choosing the equation of state $P(\rho)$ determines the model, and Eq. (4.3) can then be (numerically) solved to give the density profile $\rho(r)$.

\section{1 (non-singular) Isothermal Sphere}

The (non-singular) Isothermal Sphere is based on the equation of state of an ideal gas $P=$ $n k_{B} T$, which locally becomes

$$
P(r)=\frac{k_{B} T}{m} \rho(r),
$$

where $m$ is the mass of the constituent particle. Using Eq. (4.4) in Eq. (4.3) we have a non-linear second-order differential equation, which has to be numerically solved imposing the boundary conditions $d \rho / d r(0)=0$ and $\rho(0)=\rho_{0}$, where $\rho_{0}$ is a free parameter.

\subsection{Kang \& He models}

In [22], Kang \& He use the entropy per unit mass of an ideal gas

$$
s=\ln \left(P^{3 / 2} \rho^{-5 / 2}\right),
$$

to maximize the total entropy $S$

$$
S=\int_{0}^{\infty} 4 \pi r^{2} \rho s d r=\int_{0}^{\infty} 4 \pi r^{2} \rho \ln \left(P^{3 / 2} \rho^{-5 / 2}\right) d r
$$

subject to the constraints of conservation of total energy and the virial theorem. This procedure results in the following equation of state, with $\gamma=3 / 5$ :

$$
\rho=\lambda P+\mu P^{\gamma},
$$

We refer to this model as "Kang $\& \mathrm{He}$ ". The constants $\lambda$ and $\mu$ are related to the total mass and energy. This equation of state reduces to that of an ideal gas Eq. (4.4) for $\mu=0$ and $\lambda=m / k_{B} T$. Following a similar but different approach, [22] obtain the same equation of state, but with $\gamma=4 / 5$. We call this last model "Kang \& He 2". Inserting $\rho(r)$ from Eq. (4.7) into Eq. (4.3), gives an equation for $P(r)$, whose solution gives $\rho(r)$ by Eq. (4.7).

\subsection{DARKexp}

The DARKexp model [155, 24] is significantly different from the previous models, because it does not take into account an equation of state to use in the hydrostatic equilibrium, Eq. (4.3). Instead, it (indirectly) derives the distribution function and then determines the density profile. Let us define a dimensionless density $\tilde{\rho}=\rho / \rho_{0}$ and a dimensionless distance $x=r / a$, where $\rho_{0}$ and $a$ are scale parameters. Defining $v_{g}=\sqrt{a^{2} \rho_{0} G}$, the energy per mass $E=\Phi+v^{2} / 2$, where $\Phi$ is the gravitational potential and $v$ the velocity of the particle, can be written as

$$
\varepsilon=\varphi-\frac{1}{2} \frac{v^{2}}{v_{g}^{2}}
$$


where we defined the positive and dimensionless quantities $\varepsilon=-E / v_{g}{ }^{2}$ and $\varphi=-\Phi / v_{g}{ }^{2}$.

The DARKexp model is based on the assumption that, because dark matter halos are collisionless, after the system reaches an equilibrium, each particle retains its individual energy. Thus a Boltzmann-like function must be used, not in the distribution function $f(\varepsilon)$, but in the number of particles per unit energy $N(\varepsilon) \propto f(\varepsilon) g(\varepsilon)$, where $g(\varepsilon)$ is the density of states [25]. The model also properly deals with low occupation numbers, which results in a cutoff similar to that of King models [26]. These features imply that the number of particles per unit energy $\varepsilon$ is given by

$$
N(\varepsilon)=e^{\varphi_{0}-\varepsilon}-1,
$$

where $\varphi_{0}$ is the shape parameter representing the central potential. In order to estimate the density profile, we need to use an iterative approach [25]. We start by guessing an initial estimate of the density profile $\tilde{\rho}(x)$ and calculate the resulting potential as

$$
\varphi(x)=4 \pi\left[\frac{1}{x} \int_{0}^{x} d x^{\prime} x^{\prime 2} \tilde{\rho}\left(x^{\prime}\right)+\int_{x}^{\infty} d x^{\prime} x^{\prime} \tilde{\rho}\left(x^{\prime}\right)\right] .
$$

Next, we compute the density of states as

$$
g(\varepsilon)=16 \pi^{2} a^{3} v_{g} \int_{0}^{x_{\max }(\varepsilon)} d x x^{2} \sqrt{2[\varphi(x)-\varepsilon]},
$$

where $x_{\max }$ is such that $\varphi\left(x_{\max }\right)=\varepsilon$. We then use the $N(\varepsilon)$ defined in the model, Eq. (4.9), to compute the dimensionless distribution function

$$
f(\varepsilon)=a^{3} v_{g} \frac{N(\varepsilon)}{g(\varepsilon)} .
$$

Finally, we calculate a new $\tilde{\rho}(x)$ as

$$
\tilde{\rho}(x)=4 \pi \int_{0}^{\varphi(x)} d \varepsilon f(\varepsilon) \sqrt{2[\varphi(x)-\varepsilon]} .
$$

and iterate the process with Eq. (4.J)). We find that after about 20 iterations the model converges to a density profile independent of the initial guess. Fig. $\square$ shows all the theoretical models described.

\section{Results}

We fit the various models to the data with the help of the Minuit package [27], computing the $\chi^{2}$, i.e. the minimum value of $Q$, given by

$$
Q=\Delta_{i} V_{i j}^{-1} \Delta_{j},
$$

where $\Delta_{i}=\Sigma_{T}\left(R_{i}\right)-\Sigma_{D}\left(R_{i}\right), \Sigma_{T}\left(R_{i}\right)$ is the surface density from Eq. (ㄸ. $\mathbb{C}$ ) for a given model evaluated at radius $R_{i}, \Sigma_{D}\left(R_{i}\right)$ is data surface mass density [四] and $V_{i j}$ is the error covariance matrix

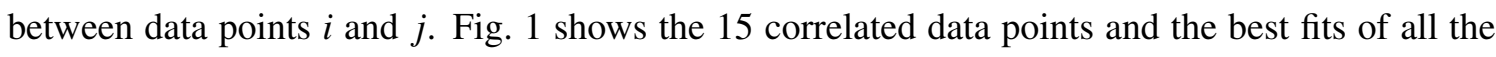
phenomenological parameters discussed above. We compare the various models using the reduced $\chi^{2}$, defined as

$$
\chi_{v}^{2}=\frac{\chi^{2}}{v}
$$

where $v=15-N_{p}$ is the number of degrees of freedom for $N_{p}$ parameters. The fitting results for the phenomenological (theoretical) models are summarized in Fig. $\square(\square)$ and Table $\square($ (】). 


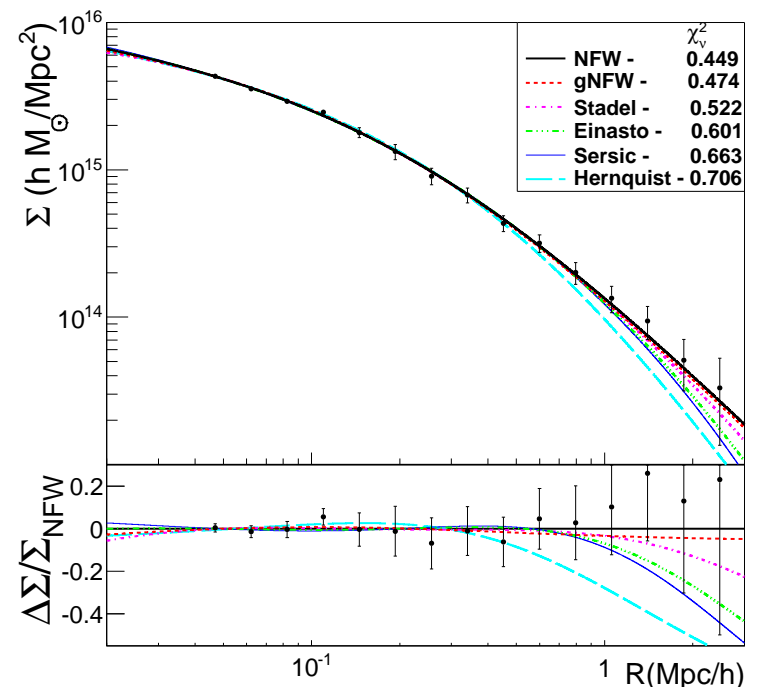

Figure 1: Best fit curves and $\chi_{v}^{2}$ for the phenomenological models studied, along with data points from [四]. The bottom panel shows the relative difference between the best fits of each model and NFW.

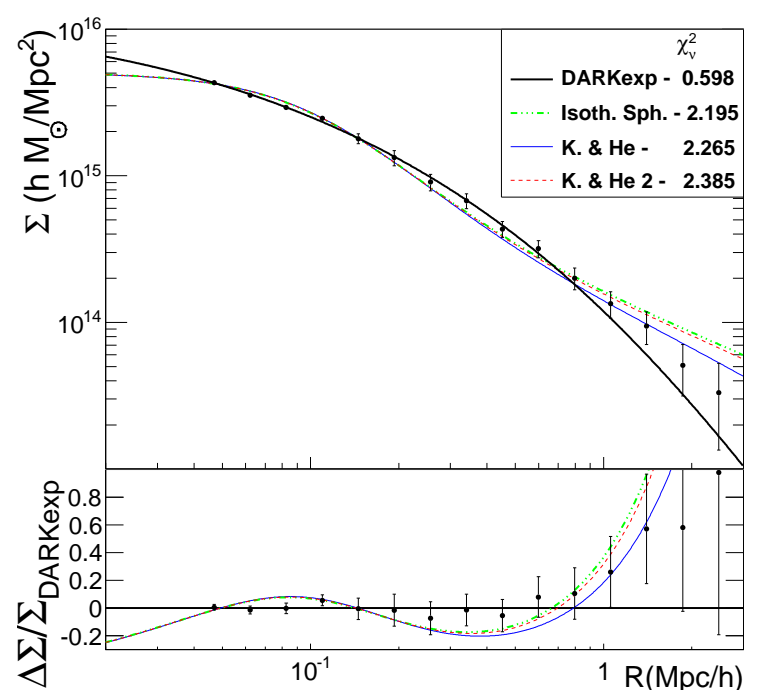

Figure 2: Best fit curves and $\chi_{v}^{2}$ for the theoretical models studied, along with data points from [四]. The bottom panel shows the relative difference between the best fits of each model and DARKexp model.

\section{Discussion}

We have used observed stacked data of 4 clusters of similar mass and redshift to study various models for cluster density profiles. We find that the best fit is obtained with the NFW model, in agreement with the results of [四]. Other phenomenological models produce similar fits with slightly larger values of $\chi_{v}$. The Isothermal Sphere and Kang \& He models produce cored profiles and give poor fits to the data. The best theoretical fit is for DARKexp, especially in the inner regions (Fig. [2). The slight disagreement on the outskirts could in principle be due to the velocity anisotropy. How- 


\begin{tabular}{|l|c|c|c|}
\hline \multicolumn{1}{|c|}{ Profile } & $N_{p}$ & $\chi_{v}^{2}$ & Shape parameter \\
\hline NFW & 2 & 0.449 & - \\
\hline gNFW & 3 & 0.474 & $\alpha=0.89 \pm 0.37$ \\
\hline Stadel & 3 & 0.522 & $\lambda=0.223 \pm 0.04$ \\
\hline Einasto & 3 & 0.602 & $n=4.31 \pm 0.75$ \\
\hline Sérsic & 3 & 0.663 & $n=2.69 \pm 0.41$ \\
\hline Hernquist & 2 & 0.706 & - \\
\hline
\end{tabular}

Table 1: Fit results for the phenomenological models. Column $N_{p}$ indicates the total number of parameters, $\chi_{v}^{2}$ shows the reduced $\chi^{2}$ and the last column shows the best estimate for the shape parameter.

\begin{tabular}{|l|c|c|c|}
\hline \multicolumn{1}{|c|}{ Profile } & $N_{p}$ & $\chi_{v}^{2}$ & Shape parameter \\
\hline DARKexp & 3 & 0.598 & $\varphi_{0}=3.24 \pm 0.48$ \\
\hline Isoth. Sph. & 3 & 2.195 & $\tilde{\lambda}=(5.62 \pm 0.15) \times 10^{4}$ \\
\hline KH & 4 & 2.265 & $\tilde{\lambda}=(5.477 \pm 0.003) \times 10^{4} ; \tilde{\mu}=6.1 \pm 0.6$ \\
\hline KH2 & 4 & 2.385 & $\tilde{\lambda}=(5.52 \pm 0.36) \times 10^{4} ; \tilde{\mu}=75 \pm 237$ \\
\hline
\end{tabular}

Table 2: Fit results for the theoretical models (columns are defined as in Table $\mathbb{W}$ ). For the shape parameters, $\tilde{\lambda}=\lambda c^{2}$ and $\tilde{\mu}=\mu\left(c^{8} / \rho_{s}\right)^{1 / 5}$, being $c$ the speed of light in vacuum.

ever, it has been shown [ [28] that this effect does not alter significantly the predicted profile. The inclusion of a 2-halo term could improve the fit of models that underestimate the outer points. Despite discrepancies at large radii, there is a global agreement between the DARKexp model and the data. A similar agreement for clusters at different masses and redshifts would indicate that the DARKexp model may provide a dynamical basis for observed dark matter density profiles.

\section{Acknowledgments}

We thank K. Umetsu for providing the cluster data and useful discussions, G. Mamon for critical reading of the manuscript, D.-B. Kang for suggestions and L. L. R. Williams for discussions that helped us reproduce the predictions of the DARKexp model. This work is supported by CNPq, CAPES and FAPESP agencies. LJBS also thanks the support of the DSU 2012 organizers.

\section{References}

[1] K. Umetsu, T. Broadhurst, A. Zitrin et al, ApJ 738 (2011) 41 [arXiv: 1105.044 ].

[2] F. Zwicky, Helv. Phys. Acta 6 (1933) 110-127.

[3] A. Bosma, PhD Thesis - Groningen University (1978)

[4] A. Bosma, P. C. van der Kruit, $A \& A 79$ (1979) 281-286.

[5] V. C. Rubin, W. K. Ford \& N. Thonnard, ApJ 225 (1978) L107-L111.

[6] V. C. Rubin, W. K. Ford \& N. Thonnard, ApJ 238 (1980) 471-487. 
[7] S. W. Allen, R. W. Schmidt \& A. C. Fabian, MNRAS 334 (2002) L11 [arXiv:astro-ph/0205007].

[8] A. Vikhlinin, A. Kravtsov, W. Forman et al, ApJ 640 (2006) 691-709 [arXiv : 0507092 ].

[9] D. Clowe, M. Bradac, A. H. Gonzalez et al, ApJ 648 (2006) L109 [arXiv:astro-ph/ 0608407 ].

[10] N. Jarosik, C. L. Bennett, J. Dunkley et al, ApJS 192 (2011) 14 [arXiv: 1001.4744 ].

[11] V. Springel, S. D. M. White, A. Jenkins et al, Nature 435 (2005) 629-636 [a st ro-ph/ 0504097 ].

[12] J. F. Navarro, C. S. Frenk \& S. D. M. White, ApJ 462 (1996) 563 [arXiv:astro-ph/9508025].

[13] J. E. Taylor \& J. F. Navarro, ApJ 563 (2001) 483-488 [arXiv:astro-ph/ 0104002 ].

[14] D. Lynden-Bell, MNRAS 136 (1967) 101.

[15] J. Hjorth \& L. R. Williams, ApJ 722 (2010) 851-855 [arXiv: 1010 . 0265].

[16] K. Umetsu, T. Broadhurst, A. Zitrin et al, ApJ 729 (2011) 127 [arXiv: 1011.3044 ].

[17] L. Hernquist, ApJ 356 (1990) 359-364.

[18] H. Zhao, MNRAS 278 (1996) 488 [astro-ph/9509122].

[19] J. F. Navarro, A. Ludlow, V. Springel et al, MNRAS 402 (2010) 21-34 [a rXiv: 0810 . 1522 ].

[20] J. L. Sérsic, Boletin de la Asociacion Argentina de Astronomia La Plata Argentina 6 (1963) 41.

[21] J. Stadel, D. Potter, B. Moore et al, MNRAS 398 (2009) L21 [arXiv : 0808 . 2981].

[22] D.-B. Kang \& P. He, A\& A 526 (2011) A147 [arXiv: 1012.1003 ].

[23] D.-B. Kang \& P. He, MNRAS 416 (2011) 32-37 [arXiv : 1104 . 3945].

[24] L. R. Williams \& J. Hjorth, ApJ 722 (2010) 856-861 [arXiv: 1010 . 0266].

[25] J. Binney, MNRAS 200 (1982) 951-964.

[26] I. R. King, AJ 71 (1966) 64-75.

[27] F. James and M. Roos, Computer Physics Communications 10 (1975) 343-367.

[28] L. R. Williams, J. Hjorth \& R. Wojtak, ApJ 725 (2010) 282-287 [arXiv: 1010.0267 ]. 